\title{
Reproducing segregation and particle dynamics in Large Eddy Simulation of particle-laden flows
}

\author{
Roxane Letournel*1,2,3 Frédérique Laurent $^{1,2}$, Marc Massot ${ }^{3}$, Aymeric Vié Me $^{1,2}$ \\ ${ }^{1}$ Laboratoire EM2C, CNRS, CentraleSupélec, Université Paris-Saclay, Gif-sur-Yvette, France \\ ${ }^{2}$ Fédération de Mathématiques de CentraleSupélec, CNRS FR-3487, CentraleSupélec, \\ Université Paris-Saclay, Gif-sur-Yvette, France \\ ${ }^{3}$ CMAP, École Polytechnique, Institut Polytechnique de Paris, Palaiseau, France \\ ${ }^{*}$ Corresponding author email: roxane.letournel@centralesupelec.fr
}

\begin{abstract}
Lagrangian simulations are today widely used for simulating aeronautical chambers. The way droplets are spatially distributed strongly affects the combustion, and accurate modeling and simulation strategies are required. The objective of the present contribution is to investigate how to correctly reproduce preferential concentration in Large Eddy Simulation (LES) of particleladen flows. Looking for a way to recover the DNS statistics, we highlight that stochastic models can fail in retrieving the tracer limit for non-inertial particles. We suggest a new strategy in the spirit of kinematic modeling of turbulence, which makes use of a random field with enforced divergence-free condition and spatial and temporal correlations. We show that the model can retrieve some Lagrangian statistics and in particular, particle segregation. We also suggest another approach for the LES particle model, based on retrieving not DNS statistics but filtered DNS statistics. We show that in this case, stochastic models can be relevant and appropriate.
\end{abstract}

\section{Keywords}

Particle dynamics, turbulence, segregation, stochastic modeling, kinematic simulations

\section{Introduction}

The Large Eddy simulation (LES) is widely used to understand and predict turbulent particleladen flows. The most classical way consists in combining a single-phase LES model to describe the gas phase, with a LES model for the particulate phase.The objective of a LES particle model is to recover the DNS statistics and macroscopic trends, such as two-way coupling between the spray and the gas phase [1]. Indeed, the retro-coupling can occur at small scales of the flow, and the LES simulation affects not only the particle phase field, but also the one of the carrier phase because of the missing interactions at small scales of the flow with particles. Models have been proposed in the literature (see the review of [2]), that can be classified into two categories:

- Structural models that aims at reconstructing the subgrid scales of the flow. Among them are the approximate deconvolution models (ADM), the fractal interpolation or kinematic simulations [3]. This approach is based on the reconstruction of the field from a Eulerian point of view.

- Stochastic models $[4,5,6,7]$, the objective of which is to mimic the effect of subgrid-scale flow on particle paths using additional random process on Lagrangian quantities.

In Ref. [8], the authors investigated the use of stochastic Langevin models. They concluded that they could produce the right amount of particle kinetic energy and any one-point statistics by construction. Models reproducing intermittency have also been developed $[9,10,11]$. However, they all assume a Markovian nature of velocity histories and do not enforce any spatial correlations: very close particles can see very different noises. They suggested that two-points 
statistics [12] can only be found in structural models to ensure particle-fluid and particle-particle velocity correlations. For large-inertia particles, the diffusion of stochastic models can replace the randomizing effect of small scales. However, those models cannot recreate the small-scale patterns solely based on coarse subgrid information for small-inertial particles.

This motivates the need for a structural approach to use more information about how real turbulence is known to behave, in particular its spatial and temporal organization. Among them, the kinematic simulation (KS) was widely studied in the literature [13, 3, 14]: it consists of a superposition of sinus and cosinus functions, such as Fourier series synthesis:

$$
u_{f}^{\star}(x, t)=\sum_{n=1}^{N} a_{n} \cos \left(\kappa_{n} x+\omega_{n} t\right)+b_{n} \sin \left(\kappa_{n} x+\omega_{n} t\right)
$$

Spatial and weight coefficients $a_{n}, b_{n}$ and $\kappa_{n}$ are chosen to match a given energy spectrum and to create a divergence-free flow. Temporal coefficients are meant to represent the eddy turnover time, but [3] found that the best fit to retrieve Lagrangian statistics such as the velocity autocorrelation is $\omega_{n}=0$. In this case, results show satisfying agreement between DNS and KS segregation, especially for particles with high Stokes numbers.

In our opinion, there are some limitations to the use of such a kinematic simulation. A frozen fluid is not an appropriate turbulence model: without sweeping, the nature of particle clustering is fundamentally changed. The lack of temporality could also explain the difficulty in mapping Eulerian and Lagrangian scales, a problem raised in the conclusion of Murray and co-workers [3].

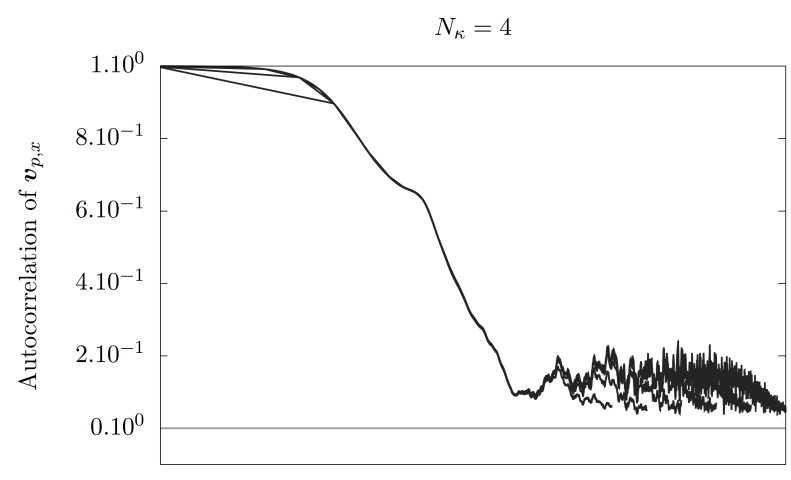

Figure 1. Auto-correlation of the velocity of fluid parcels along theur trajectory on a kinematic simulation of fluid (Figure from [15]).

In his thesis [15], Mercier investigated KS and he pointed out the divergence of the velocity autocorrelation of tracers $R_{p}^{L}(\tau)=\left\langle v_{p}(t) v_{p}(t+\tau)\right\rangle /\left\langle v_{p}(t)^{2}\right\rangle$, reported in Fig. 1. Normally, particle velocities should be completely uncorrelated after a very long time, and the autocorrelation should tend towards 0 . The oscillating and divergent behavior of the Lagrangian statistics could be attributed to the non-compactness of the cosinus and sinus modes.

Therefore, we are exploring a different approach for kinematic simulation, and we present a new synthetic fluid with the following specifications:

- C1 The synthetic fluid is spatially correlated with compact modes

- C2 It is a divergence-free flow, to recover the tracer limit

- C3 It can reproduce any energy spectrum

- C4 The temporal correlation can be fitted to map Eulerian scales with Lagrangian ones

- C5 Lagrangian one and two-point DNS-like statistics can be recovered 
- C6 The flow must be compatible with LES framework

- C7 The method should be computationally affordable (of the order of magnitude of the LES complexity).

\section{A new kinematic simulation: Capturing DNS Statistics}

Structural models have the advantage of being able to produce a spatial structure of the flow field. Segregation is strongly related to the existence of small scale and large scale coherent structures, which are not present in stochastic modeling. In the following, we propose to develop a synthetic field as a basis for structural models. The objective is to construct a velocity field $u(t, x)$ without solving the Navier-Stokes equation but keeping all the turbulence statistics of interest such as the Kolmogorov time or the integral time scales. To this aim, we will decompose the problem into two sub-problems: space reconstruction and time evolution.

\section{Space reconstruction with compact modes (C1, C2, C3)}

For the space reconstruction, we generate the velocity field using the following technique:

- We first generate three Gaussian random fields $A_{x}, A_{y}, A_{z}$, over which we impose a spatial correlation through applying a spatial filter (here chosen as Gaussian). The size of the filter $\sigma_{f}$ determines the length of correlation $\ell_{c}$ of the field. As opposed to the Fourier series of classic KS, the correlation length is compact because it is directly generated with the Gaussian filter of finite size.

- We generate a divergence-free 3D flow using $v_{\ell_{c}}(x)=\nabla \times A$.

- By superposing velocity fields with varying filter sizes, we can control the turbulence spectrum. Figure 2(a) shows that each subfield $v_{\ell_{c}}$ produces an energy spectrum with a maximum located at the correlation length of the field $\kappa_{\max } \sim \ell_{c}^{-1}$. With an adapted linear combination, it is possible to produce an energy spectrum with slope $-5 / 3$ in Fig. 2(b). We normalize it so that its variance is 1 . An example of the velocity and vorticity fields obtained are presented in Fig. 2(c) and Fig. 2(d).

The generated field $v(x)=\sum_{n=1}^{N_{m}} \alpha_{n} v_{\ell_{n}}(x)$ is therefore spatially-correlated (with compact modes), divergence-free, and its energy spectrum is controlled with the number of modes (subfields) and the range of correlation lengths: from the largest scale $L=\ell_{1}$, to the smallest $\eta=\ell_{N_{m}}$. This gives a frozen flow and we add a temporal correlation in the following section.

\section{Time evolution for Eulerian Lagrangian mapping (C4 and C5)}

Compared to kinematic simulation, we do not have an analytic expression in which we can include time evolution in a straightforward manner. To introduce a temporal correlation, we decided to use a stochastic equation in which we replace the usual Gaussian noise $d W_{t}$ by the spatially correlated field $v(x)$ introduced in previous section. Each node of the Eulerian grid follows a Langevin equation, since the noise $v(x)$ is Gaussian, of variance 1 and we generate a new field $v_{t}(x)$ at each time step (so increments are uncorrelated) and inject it in this equation:

$$
d u_{t}(x)=-\frac{1}{T_{E}} u_{t}(x) d t+\sqrt{\frac{2 \sigma_{u}^{2}}{T_{E}}} v_{t}(x) \sqrt{d t}
$$

Because $v_{t}(x)$ is spatially correlated, $u_{t}(x)$ keeps this spatial correlation and neighboring nodes in the grid will have a similar evolution. $T_{E}$ is the Eulerian correlation time, and it controls the speed of change of the flow. When large, temporal increments $d u_{t}(x)$ tend towards 0 and the flow becomes frozen. On the other hand, if $T_{E}$ is small, the velocity field changes very fast, and 


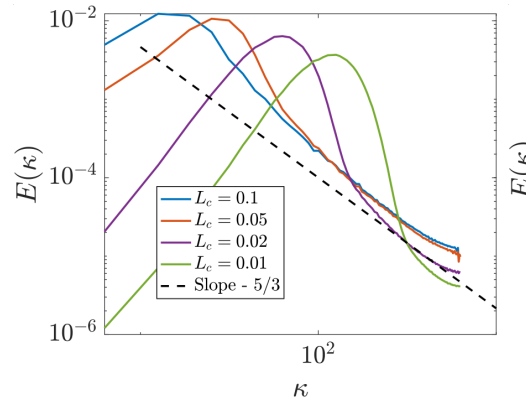

(a) Unique mode

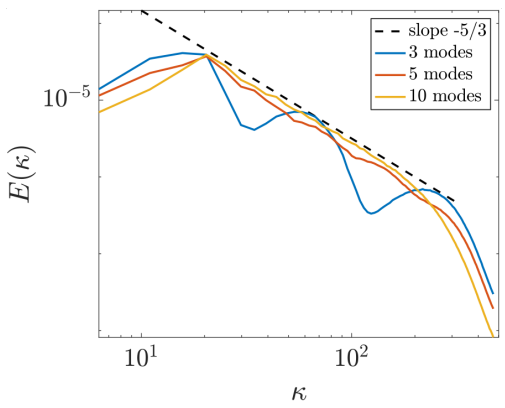

(b) Several modes

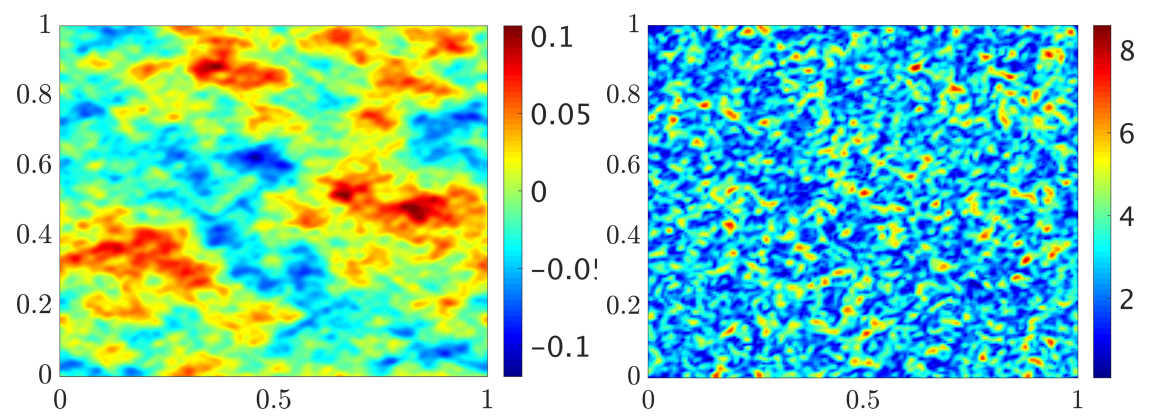

(c) Velocity component with 10 modes

(d) Vorticity with 10 modes

Figure 2. Spatially-correlated random field with zero divergence: (a) influence of the filtering length $L_{c}$ for a unique field, (b) superposition of different random fields to control the turbulence spectrum, (c) example of the generated velocity field and (d) vorticity field. All parameters are dimensionless.

the relaxation towards the perturbation $v_{t}(x)$ is immediate. The coefficient $\sqrt{2 \sigma_{u}^{2} / T_{E}}$ before the noise ensures the stationarity of the Langevin Equation (and therefore of the flow statistics), with a given variance $\sigma_{u}^{2}$. We recall that $\sigma_{u}$ gives the intensity of the forcing and is related to the turbulent kinetic energy $k=3 / 2 \sigma_{u}^{2}$. The Kolmogorov time-scale is related to the fluid variance and the lengthscales in $v(x): \tau_{\eta} \sim \ell_{c} / \sigma_{u}$, where $\ell_{c}$ is the mean correlation length of the flow.

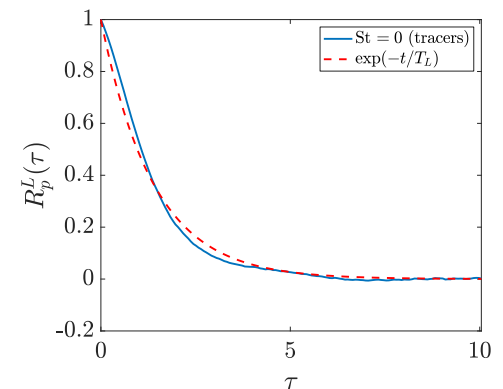

(a) Lagrangian velocity autocorrelation

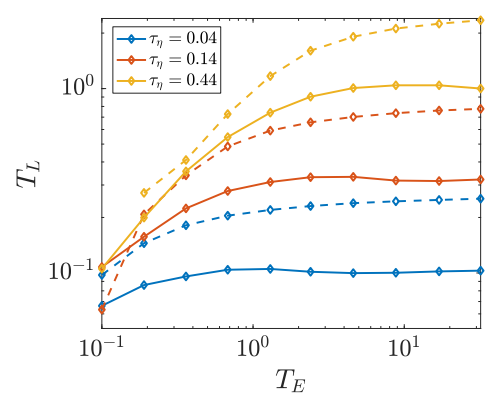

(b) Simple line: one mode, dotted line: 3 modes

Figure 3. One-point Lagrangian statistics: Lagrangian velocity autocorrelation fitted with an exponential function $\left(T_{E}=3\right.$ and $\left.\tau_{\eta}=0.7\right)$ (a) and Lagrangian integral time scale of turbulence as a function of $T_{E}$ for different Kolmogorov time scales $\tau_{\eta}$ and different number of modes (b). All parameters are dimensionless.

We can show that the introduction of the temporal evolution provides parameters to control Lagrangian scales, such as $T_{L}=\int_{0}^{\infty} R_{p}^{L}(\tau) d \tau$, the integral time scale. As opposed to the diverging autocorrelation with classic KS in Fig. 1, the one obtained in Fig. 3(a) converges towards 0 with an exponential decrease. Figure 3(b) shows the dependency of $T_{L}$ with $T_{E}$ and it is clear, even with a single mode, that increasing the Eulerian correlation time of the flow also raises the integral Lagrangian time to a maximum, which is obtained for frozen turbulence. 
Moreover, keeping the same $\tau_{\eta}$ but adding more scales in the inertial range with additional modes can also increase $T_{L}$ (dotted lines in Fig. 3(b)).

An interesting perspective is to imagine having different temporal evolution for each sub-field $v_{\ell_{n}}$ for which an adapted $T_{E}$ could be introduced.

Those additional parameters regarding an Eulerian temporal evolution can be adapted and fitted to retrieve Lagrangian one-point statistics. In the next section, results for two-point statistics are discussed.

\section{Particle segregation in the synthetic turbulence}

Table 1. Parameters of the fluid model used as a carrier phase. All parameters are dimensionless.

\begin{tabular}{cccccc}
\hline$N_{m}$ & $\ell_{c}$ & $\sigma_{u}^{2}$ & $\tau_{\eta}$ & $T_{E}$ & $T_{L}$ \\
\hline 1 & 0.03 & 0.01 & 0.4 & 4 & 1 \\
3 & $0.01,0.03,0.1$ & 0.01 & 0.4 & 4 & 2
\end{tabular}

Now we have a time and space varying field, and we can use it as a carrier phase for the dynamics of particles, which is the aim of the present contribution. Table 1 shows the model parameters chosen to simulate the synthetic fluid.

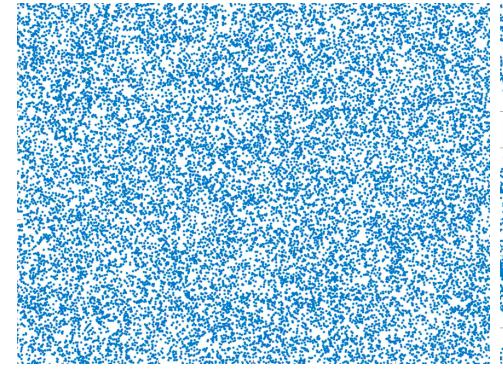

(a) St $=0$

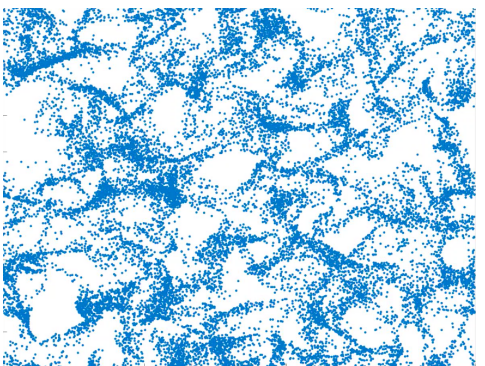

(b) $\mathrm{St}=2$

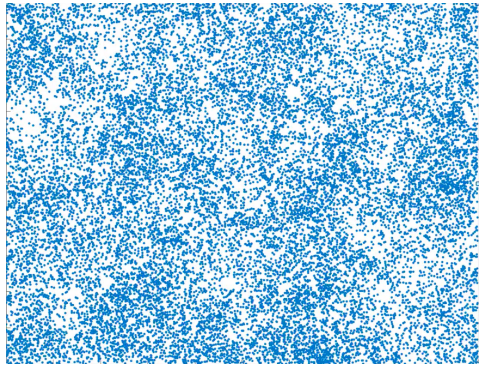

(c) $\mathrm{St}=10$

Figure 4. Particles in a slice of the 3D synthetic turbulence for different Stokes numbers at time $t=6$ for $\tau_{\eta}=0.44$ and one mode $\left(N_{m}=1\right.$ in Table 1$)$. All parameters are dimensionless.

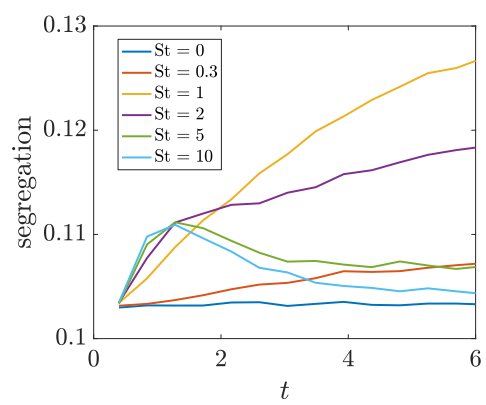

(a) Segregation with one mode

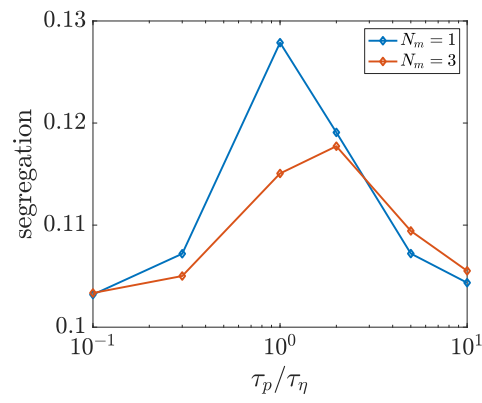

(b) Segregation with 1 and 3 modes

Figure 5. Temporal evolution of segregation (variance of the particle number density distribution) of particles interacting with the kinematic fluid of one mode (a) and segregation at time $t=6$ for one and 3 modes (b). All parameters are dimensionless.

We investigate the effect of Stokes number on the segregation, even with a single mode. First, we show that we retrieve the tracer limit for zero-inertia particles for which no segregation is expected. In Fig. 4(a), thanks to the divergence-free, the tracers are not preferentially concentrated and fill the space homogeneously. Particles with Stokes number close to 1, in Fig. 4(b), 
are preferentially concentrated by the reconstructed scales of the synthetic flow, and the segregation increases with time, as shown in Fig. 5(a). Particles with high Stokes number (Fig. 4(c)) are first ejected out of vortices: the segregation rises at the beginning of the simulation, but their large inertia prevents them from evolving with the small scales, and particle trajectory crossing occurs shortly after.

The temporal trends of Fig. 5(a) are similar to what is expected for particles in real turbulence and allow us to conclude that our field is appropriate as a surrogate for Navier-Stokes simulation to predict particle segregation in turbulence. Moreover, Fig. 5(b) shows that changing the number of modes in the synthetic fluid can also adjust the intensity of the segregation quantitatively. This additional information could be helpful to fit the segregation level to the DNS.

\section{Compatible with LES framework (C6)}

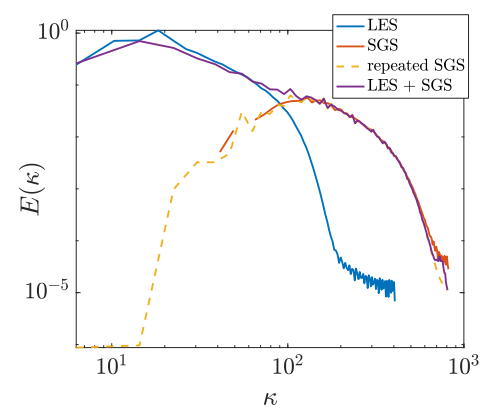

(a) Energy spectrum

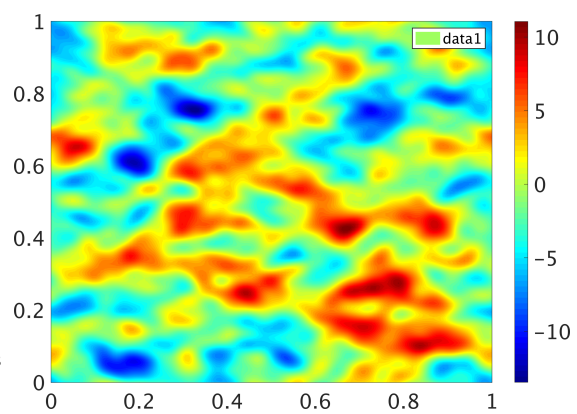

(b) LES

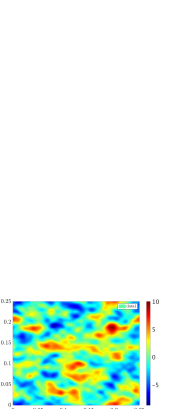

(c) SGS

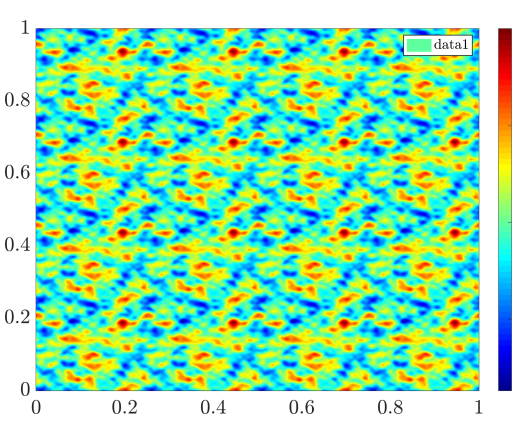

(d) Repeated SGS

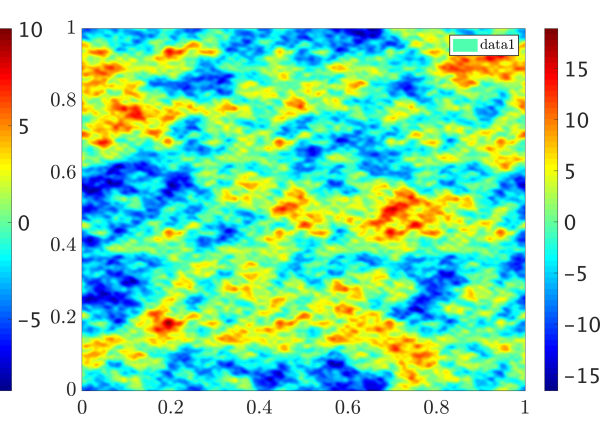

(e) LES + SGS

Figure 6. Energy spectrum of LES and LES combined with the synthetic flow(a), snapshot of LES velocity field (a), synthetic fluid with small scales (b), repeated patterns of the synthetic fluid (c) and LES and synthetic fluid combined (d). All parameters are dimensionless.

The versatility of this method due to the choice of the characteristic lengths allows us to adapt the generated field according to the LES characteristics easily. Indeed, the synthetic field can be generated to complete only the small missing scales and thus reconstruct a complete DNSlike spectrum. This is illustrated in Fig. 6(a) where a LES spectrum is completed with a sub-grid flow.

\section{Dimensionality reduction (C7)}

However, generating subgrid scales everywhere leads to the same memory space as a DNS. Therefore, we investigate dimensionality reduction. Instead of generating a field on the complete refined mesh, only a part of this field is simulated on a limited area (Fig. 6(c)), and then replicated on the complete domain in a second step (Fig. 6(d)). The combination of this reproduced field with the large scales of the LES produces the field in Fig. 6(e) and reconstructs the spectrum shown in Fig. 6(a).

We are also investigating wavelet decomposition [16] because unlike Fourier series, wavelets 
maintain the compacity, and the locality of the information and compression could reduce the cost. Figure 7 compares a wavelet decomposition using Haar wavelets or Daubechies wavelets. The original field is compressed with a ratio of $1 \%$. Certainly, the choice of the wavelet mother is crucial to expect a fair representation of the initial field. The level of compression and the amount of information will also be critical.

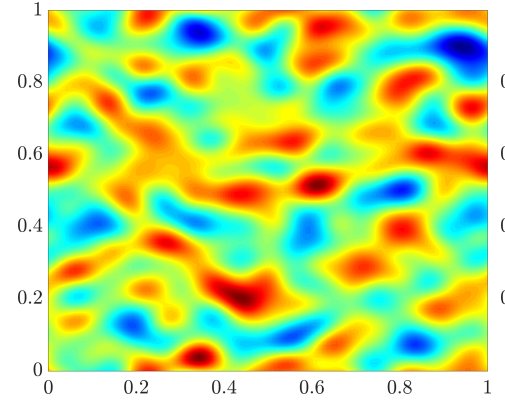

(a) Synthetic field

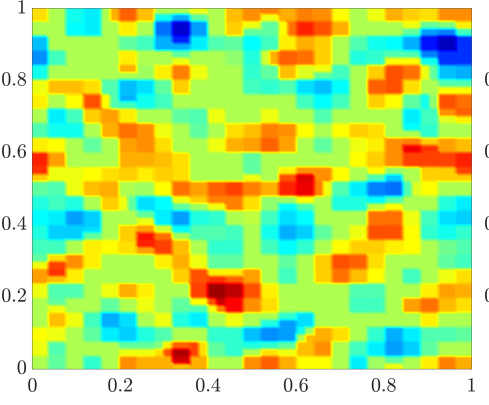

(b) Haar wavelets

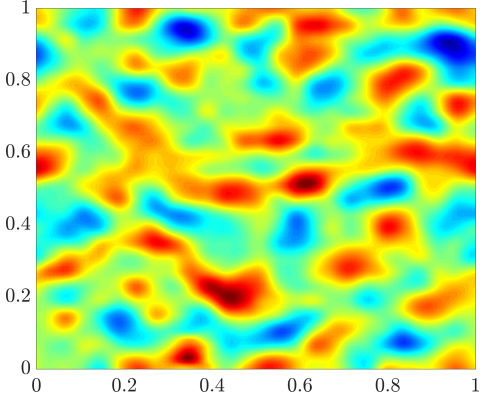

(c) Daubechies wavelets

Figure 7. Snapshot of a synthetic field (a), and the same field is decomposed using Haar wavelets (b) or Daubechies wavelets (c) with a compressing ratio of $1 \%$.

\section{Perspectives: Another strategy for capturing LES Statistics}

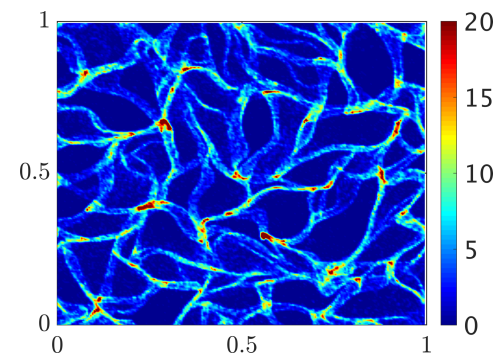

(a) DNS field

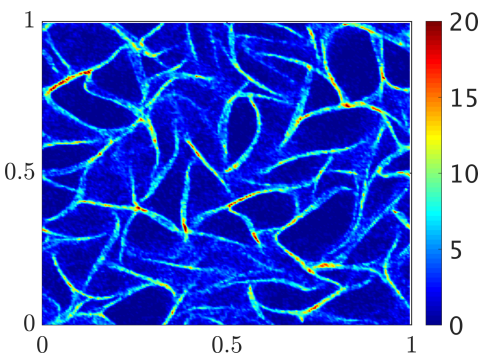

(b) LES filtered field

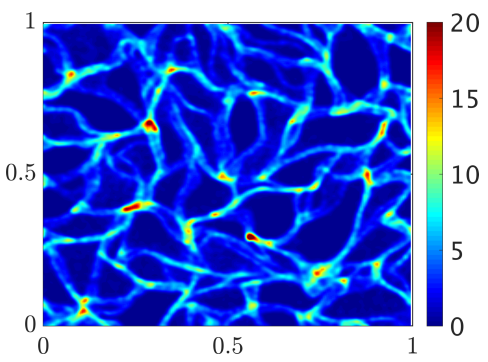

(c) filtered DNS field

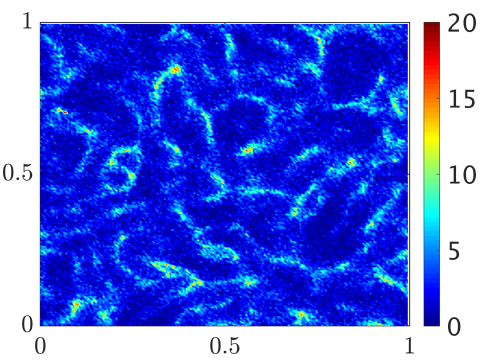

(d) LES and stochastic model

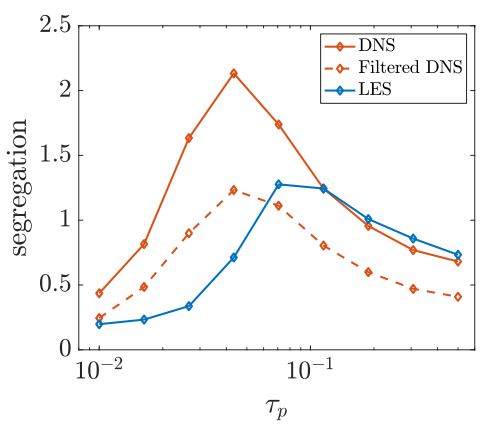

(e) Variance of the PND field

Figure 8. Particle number density field for (a) DNS, (b) LES with no model, (c) filtered PND field of DNS, (d) LES with stochastic modeling, and (e) Variance of the Probability distribution function of number of particles per bin.

In the present work, we have explored modeling strategies for LES of particle-laden flows in the scope of capturing particles dynamics. Such dynamics require a correct description of the particle localization (preferential concentration) as well as particle acceleration. Following the conclusions of [8], we would expect that stochastic models are not an acceptable choice because they lack a correct prediction of preferential accumulation. This conclusion is based on a specific strategy for validation: the authors validate LES models against the DNS statistics. However, this choice is open to discussion since one can also decide to validate the LES models 
on the filtered DNS statistics. These two options differ by the objective of the model in sight: describing the PDF or the filtered PDF.

We perform a DNS simulation of particle-laden turbulent flows, and we show the particle number density (PND) field in Fig. 8(a). The same PND field is filtered and presented in Fig. 8(c). Those PND are the two possible references to establish a LES particle model. The segregation of the filtered PND is lower: we now have excessive segregation for the LES, and adding a stochastic process permits to reduce the segregation and eventually recover the missing physics, as shown in Figs. 8(d) and 8(e).

\section{Conclusions}

Depending on LES particulate models' objective, it is possible to derive two strategies to retrieve two-points statistics such as segregation. In the attempt to recover DNS statistics, we investigate a new approach in kinematic simulation. We propose a new model, with spatial and temporal correlation which parameters can be adjusted to retrieve Eulerian and Lagrangian DNS statistics. In order to make the simulation of such fields affordable and compatible with LES, compression strategies will be considered and explored using proper orthogonal decomposition, or davelet decomposition. Perspectives, of course, include an exhaustive comparison of this technique with classic KS and against DNS simulations. Finally, in the attempt to recover filtered-DNS statistics, stochastic modeling could be very satisfying, and we are currently working on comparing them and assessing them from this perspective.

\section{Acknowledgements}

This work was supported by grants from Region lle-de-France DIM MATHINNOV. Support from the French Agence Nationale de la Recherche in the MIMETYC project (grant ANR-17-CE220003) is also acknowledged.

\section{References}

[1] Letournel, R., Laurent, F., Massot, M., and Vié, A., 2020, International Journal of Multiphase Flow, 125, p. 103233.

[2] Marchioli, C., 2017, Acta Mechanica, 228(3), pp. 741-771.

[3] Murray, S., Lightstone, M.F., and Tullis, S., 2016, Physics of Fluids, 28(3).

[4] Minier, J.P., Chibbaro, S., and Pope, S.B., 2014, Physics of Fluids, 26(11).

[5] Bini, M., and Jones, W.P., 2008, Journal of Fluid Mechanics, 614, pp. 207-252.

[6] Shotorban, B., and Mashayek, F., 2006, Journal of Turbulence, 7(7), p. N18.

[7] Zamansky, R., Vinkovic, I., and Gorokhovski, M., 2013, Journal of Fluid Mechanics, 721, pp. 627-668.

[8] Pozorski, J., and Apte, S.V., 2009, International Journal of Multiphase Flow, 35(2), pp. 118-128.

[9] Schmitt, F.G., 2003, European Physical Journal B, 34(1), pp. 85-98.

[10] Pereira, R.M., Moriconi, L., and Chevillard, L., 2018, Journal of Fluid Mechanics, 839, pp. 430-467.

[11] Letournel, R., Goudenège, L., Zamansky, R., Vié, A., and Massot, M., 2021, Submitted in Physical Review E, available on HAL and ArXiv, 1-15.

[12] Eaton, J.K., and Squires, K.D., 1991, Journal of Fluid Mechanics, 226(-1), p. 1.

[13] Kraichnan, R.H., 1970, Physics of Fluids, 13(1), pp. 22-31.

[14] Ijzermans, R.H.A., Meneguz, E., and Reeks, M.W., 2010, Journal of Fluid Mechanics, 653, pp. 99-136.

[15] Mercier, D., 2020, Large eddy simulation of coupled dispersed phase flows: a statisticallyconsistent formalism. Ph.D. thesis, Université Paris-Saclay.

[16] Farge, M., 1992, Dahan et al, pp. 1-23. 